\title{
ANATOMÍA DEL TALLO Y DE LA RAÍZ DE DOS ESPECIES DE Wilcoxia Britton \& Rose (Cactaceae) del noreste de México
}

\author{
Sofía Loza-Cornejo y Teresa Terrazas \\ Programa de Botánica, Colegio de Postgraduados, \\ Montecillo, 56230 Estado de México, México. e-mail: winchi@colpos.colpos.mx
}

\begin{abstract}
Resumen. Se estudia la anatomía del tallo y la raíz de dos especies de Wilcoxia distribuidas en el noreste de México con la finalidad de contribuir al conocimiento de las cactáceas, cotejar la ausencia de radios en su xilema secundario y comparar su anatomía con la de otros miembros de Cactoideae. Los resultados muestran que las especies de Wilcoxia presentan hipodermis uniestratificada, peridermis unidireccional, clorénquima en empalizada, ausencia de fibras en el floema y de radios en el xilema secundario. Se considera que la ausencia de radios probablemente fue un factor importante en la evolución del cámbium vascular de estas especies. Las especies difieren en algunos caracteres cuantitativos y en el tipo porosidad. En las raíces tuberosas de Wilcoxia el tejido vascular es escaso y restringido a hileras radiales, mientras que el tejido de almacenamiento se desarrolla en corteza primaria y parénquima asociado al xilema secundario. Los estudios del desarrollo de la raíz tuberosa, podrían contribuir al entendimiento de la diversidad anatómica encontrada en Cactaceae.

Palabras clave: Cactaceae, Wilcoxia, anatomía del tallo, madera sin radios, anatomía de la raíz tuberosa.

Abstract. Stem and root anatomy of two Wilcoxia species distributed in the northeast of Mexico are studied to contribute to cacti knowledgement, to conclude about their rayless wood and to compare their anatomy with that of other Cactoideae members. Results show that Wilcoxia species have unistratified hypodermis, palisade chlorenchyma, lack of fibers in phloem, and rayless wood. Raylessness probably was an important factor in the evolution of vascular cámbium in these species. Species differ in some quantitative characters and porosity type. In Wilcoxia's tuberous root, vascular tissue is scarse and restricted to radial rows, while storage tissue occurs in cortex and parenchyma associated to secondary xylem. Additional tuberous root developmental studies should contribute to understand anatomical diversity reported for Cactaceae.
\end{abstract}

Keywords: Cactaceae, Wilcoxia, stem anatomy, rayless wood, tuberous root anatomy.

a familia Cactaceae incluye especies con tallos suculentos, que van desde el tamaño pequeño con xilema secundario escasamente desarrollado, hasta formas arborescentes con xilema secundario abundante (Gibson, 1973). De las tres subfamilias, Cactoideae es la mejor representada en México (Barthlott y Hunt, 1993) e incluye géneros con diversidad de hábito, tamaño, hábitat, ecología y anatomía (Barthlott y Hunt, 1993).

México es el centro más importante de distribución de cactáceas y la mayor parte de sus especies habitan en regiones áridas y semiáridas del país (Arias, 1993; Hernández y Godínez, 1994). Sin embargo, hasta la fecha, los estudios anatómicos realizados en géneros endémicos a México son escasos (Altesor et al.,
1994; Boke, 1951, 1959; Eggli, 1984; Flores-Vindas, 1973; Gibson 1973, 1978a; Gibson y Horak, 1978; Hernández-García y García-Villanueva, 1991; Orozco, 1987; Pimienta et al., 1993). El género Wilcoxia fue seleccionado como sistema de estudio porque es poco conocido anatómicamente. Se tiene información sólo para algunos caracteres de la epidermis (Eggli, 1984) y algunos del xilema secundario (Gibson, 1973). En ese trabajo Gibson estudió W. albiflora Backeberg y sugiere examinar las otras especies de Wilcoxia para ver si los radios en el xilema secundario también están ausentes.

El género Wilcoxia sensu Bravo-Hollis (1978) incluye seis especies presentes en el desierto Sonorense o Chihuahuense. En el noreste de México se distribuyen 
Cuadro 1. Localidades de recolecta de las especies de Wilcoxia

\begin{tabular}{lll}
\hline Especies & Localidad & Individuos recolectados \\
\hline W. poselgeri & Progreso-Monclova, Coah. & 4 (Terrazas 325-328) \\
W. poselgeri & Planicie Saltillo-Torreón, Coah. & 1 (Sánchez-Mejorada 2567)* \\
W. poselgeri & Carr. Monterrey-Laredo, N. L. & 1 (Bravo-Hollis 3228)* \\
W. tamaulipensis & Presa de la Rosa, Coah. & 2 (Terrazas 323-324)) \\
W. tamaulipensis & La Reforma, Tamps. & 4 (Terrazas y Loza 319-322) \\
W. tamaulipensis spp. waldeisii & El Huizache, S. L. P. & 2 (Hernández et al. H-2688, C-938) \\
\hline
\end{tabular}

* Material tomado de MEXU.

solamente dos especies, W. poselgeri (Lemaire) Britton y Rose y W. tamaulipensis Werdermann. Cabe señalar que Lange (1995) reconoce tres subespecies bajo el nombre Echinocereus tamaulipensis, aceptando la clasificación de Taylor (1985). Dichas subespecies son E. tamaulipensis ssp. tamaulipensis (Werdermann) M. Lange, E. tamaulipensis ssp. deherdtii $\mathrm{M}$. Lange y $E$. tamaulipensis ssp. waldeisii (E.Haugg) M. Lange. A la fecha realizamos un estudio sistemático en el género Echinocereus con objeto de encontrar evidencias adicionales que permitan entender sus límites.

Las especies de Wilcoxia son de talla pequeña, no mayores de un metro (figura 1); tienen tallos de 6 a $10 \mathrm{~mm}$ de diámetro con 8 a 10 costillas poco elevadas, y en las areólas se presentan de 9 a 20 espinas radiales y de 1 a 10 centrales. Son plantas erectas o débilmente escandentes con crecimiento secundario (Bravo-Hollis, 1978; Britton y Rose, 1963; Buxbaum, 1973), que se distinguen por tener una raíz principal con ramificaciones más o menos numerosas. Algunas raíces de segundo o tercer orden presentan tubérculos fusiformes o cilindroides que se denominan raíces tuberosas o fasciculado-tuberosas (figura $1)$, con peridermis comúnmente de color obscuro.

El presente estudio es una contribución al conocimiento de la anatomía de las especies de Wilcoxia distribuidas en el noreste de México, pretende cotejar la ausencia de radios en el género y comparar las características anatómicas de dichas especies con las de otros miembros de Cactoideae.

\section{Materiales y métodos}

En cada localidad se recolectaron de uno a cuatro individuos de las especies de Wilcoxia en los estados de Coahuila, San Luis Potosí y Tamaulipas (cuadro 1). El material herborizado de dichas colectas se depositará en CHAPA y MEXU. Muestras adicionales provienen de material removido de ejemplares de herbario depositados en MEXU (cuadro 1). También se remo- vió material de la parte basal del tallo de un ejemplar de W. albiflora (P. Fisher, recolectado en Sonora el 27 de abril de 1962, MEXU) con objeto de comparar la anatomía del xilema secundario de esta especie con las estudiadas del noreste de México. Del material recolectado se tomaron muestras de la parte apical, media y basal del tallo y de la raíz no tuberosa y tuberosa de cada individuo, y se fijaron en FAA (Berlyn y Miksche, 1976) en el campo. El material removido de ejemplares de herbario se hidrató con agua destilada y posteriormente se fijó con FAA. Parte del material fijado se colocó en etilendiamina al $4 \%$ para ablandarlo (Carlquist, 1982). Este material se deshidrató en un cambiador automático Fisher; se incluyó en parafina y fue seccionado con un micrótomo rotatorio a $15 \mu \mathrm{m}$ de grosor. Los cortes se tiñeron con safranina-verde fijo y se montaron con resina. Para las impresiones epidérmicas se utilizó una mezcla de pasta (Xantopren VL) y activador líquido (Optosil Xantopren Activator, Bayer). Para el estudio del xilema secundario se hicieron cortes de las caras transversal, tangencial y radial de 20 a $25 \mu \mathrm{m}$ de grosor con un micrótomo de deslizamiento. Los cortes se tiñeron con safranina al $0.5 \%$ en etanol al $95 \%$ y se montaron también con resina. La disociación de la madera se hizo de acuerdo a Berlyn y Miksche (1976). La medición de los caracteres anatómicos se realizó con un ocular micrométrico y se midieron 25 unidades por individuo para las células de la epidermis, hipodermis y del xilema secundario. Para los caracteres cuantitativos se obtuvieron algunos parámetros de la estadística descriptiva, media, desviación estándar, mínimo y máximo. La existencia de diferencias significativas para los caracteres cuantitativos entre especies se buscó mediante la estadística de T-Student (SAS, 1989). Esta no se practicó a las variables grosor de la cutícula, densidad de vasos $/ \mathrm{mm}^{2}$ y diámetro tangencial de vasos. En el grosor de la cutícula, por ser el número de observaciones menor a lo fijado, y en la densidad de vasos y el diámetro de éstos por 
no ser comparables los diferentes tipos de porosidad (Terrazas, 1994). La terminología empleada para la descripción de la epidermis se basa en Wilkinson (1979) y para la descripción del xilema secundario en las recomendaciones del Comité de la Asociación Internacional de Anatomistas de la Madera (IAWA 1964, 1989). La terminología para el tipo de punteaduras intervasculares sigue a Carlquist (1988).

\section{Resultados}

\section{Descripción anatómica del tallo}

Cutícula y epidermis. La cutícula tiene un grosor $<1 \mu \mathrm{m}$ en $W$. poselgeri y más gruesa en $W$. tamaulipensis (cuadro 2). Las células de la epidermis son rectangulares (figuras 2 y 3 ) y miden $24 \pm 5 \mu \mathrm{m}$ de ancho por $53 \pm 8 \mu \mathrm{m}$ de largo en $W$. tamaulipensis y $26 \pm 8 \mu \mathrm{m}$ de ancho por $50 \pm 11 \mu \mathrm{m}$ de largo en $W$. poselgeri (cuadro 2). Estas son lisas y en vista superficial tienen paredes anticlinales sinuosas. Los estomas se encuentran principalmente en las depresiones de las costillas, son del tipo paracítico y sus células oclusivas están al mismo nivel que las células epidérmicas adyacentes.

Peridermis. Se presenta inicialmente en forma de parches en la superficie de las costillas y se desarrolla progresivamente hacia las depresiones de éstas, hasta cubrir completamente el tallo. El felógeno se origina de células epidérmicas que mediante divisiones periclinales forman felema. El felema se distingue por tener una capa de células con pared no lignificada cercana al felógeno, seguida hacia el exterior por dos a cinco estratos de células rectangulares colapsadas con paredes lignificadas. En la parte más basal del tallo la peridermis tiene capas alternantes de células con pared lignificada y células de pared no lignificada (figura 4). Las células de pared no lignificada están colapsadas radialmente y es difícil distinguir el número de estratos; mientras que las células de pared lignificada, esclereidas, conservan su forma rectangular y forman bandas discontinuas con un espesor de dos a cuatro células (figura 4).

Hipodermis. Tiene un estrato de células de pared delgada con un grosor que varía de $20 \pm 3 \mu \mathrm{m}$ en $W$. poselgeri a $30 \pm 6 \mu \mathrm{m}$ en $W$. tamaulipensis (cuadro 2). Esta hipodermis se interrumpe por cámaras subestomáticas que penetran hasta las células corticales adyacentes.

Corteza primaria. Tiene un espesor de hasta $216 \mu \mathrm{m}$ y está formada por dos regiones, clorénquima y reservante (figuras 2,3 y 5 ). Las células del clorénquima son las más cercanas a la hipodermis, tienen forma rectangular o cuadrada y forman hileras radiales perpendiculares a la superficie de las costillas (figura 3). Las células de parénquima no fotosintético de la corteza interna son de forma más o menos isodiamétrica y pueden o no presentar granos de almidón. En ambas regiones de la corteza se encuentran haces vasculares, células de mucílago y cristales (figura 5). Los haces vasculares corticales a lo largo del tallo son colaterales; constituidos por un número variable de vasos y por elementos de tubo criboso de forma angulosa y células acompañantes más o menos triangulares (figura 6). Cabe destacar que los haces vasculares corticales de la parte basal del tallo presentan crecimiento secundario. Este crecimiento secundario se

Cuadro 2.Valores de la media, una desviación estándar y (el mínimo y el máximo) de los caracteres anatómicos cuantitativos del tallo de las especies de Wilcoxia estudiadas. Las medias con la misma letra no son estadísticamente diferentes entre especies ( $p>|\mathrm{T}|$ 0.0001).

\begin{tabular}{lll}
\hline Carácter & W. poselgeri & W. tamaulipensis \\
\hline Ancho de células epidérmicas $(\mu \mathrm{m})$ & $26 \pm 8^{\mathrm{a}}(16-42)$ & $24 \pm 5^{\mathrm{a}}(18-50)$ \\
Largo de células epidérmicas $(\mu \mathrm{m})$ & $50 \pm 11^{\mathrm{a}}(30-80)$ & $53 \pm 8^{\mathrm{a}}(22-74)$ \\
Grosor de cutícula $(\mu \mathrm{m})^{*}$ & $<1.0$ & $4.0 \pm 0.5$ \\
Grosor de la hipodermis $(\mu \mathrm{m})$ & $20 \pm 3^{\mathrm{a}}(15-26)$ & $30 \pm 6^{\mathrm{b}}(21-42)$ \\
Diámetro de cristales esferoidales $(\mu \mathrm{m})$ & $62 \pm 15^{\mathrm{a}}(48-115)$ & $149 \pm 20^{\mathrm{b}}(113-195)$ \\
Densidad de vasos/mm ( $^{2 *}$ & $6 \pm 2(3-10)$ & $10 \pm 3(5-20)$ \\
Longitud elemento de vaso $(\mu \mathrm{m})$ & $155 \pm 26^{\mathrm{a}}(106-256)$ & $181 \pm 29^{\mathrm{b}}(120-264)$ \\
Diámetro tangencial del vaso $(\mu \mathrm{m})^{*}$ & $15 \pm 3(10-20)$ & $17 \pm 4(14-22)$ \\
Longitud de fibra $(\mu \mathrm{m})$ & $313 \pm 50^{\mathrm{a}}(10-20)$ & $316 \pm 43^{\mathrm{a}}(232-408)$ \\
Diámetro de fibra $(\mu \mathrm{m})$ & $15 \pm 2^{\mathrm{a}}(10-20)$ & $15 \pm 3^{\mathrm{a}}(12-20)$ \\
Grosor de la pared de la fibra $(\mu \mathrm{m})$ & $3.6 \pm 0.6^{\mathrm{a}}(2.4-4.8)$ & $3.0 \pm 0.6^{\mathrm{b}}(2.4-7.2)$ \\
\hline
\end{tabular}

* No se realizó la T-Student. 


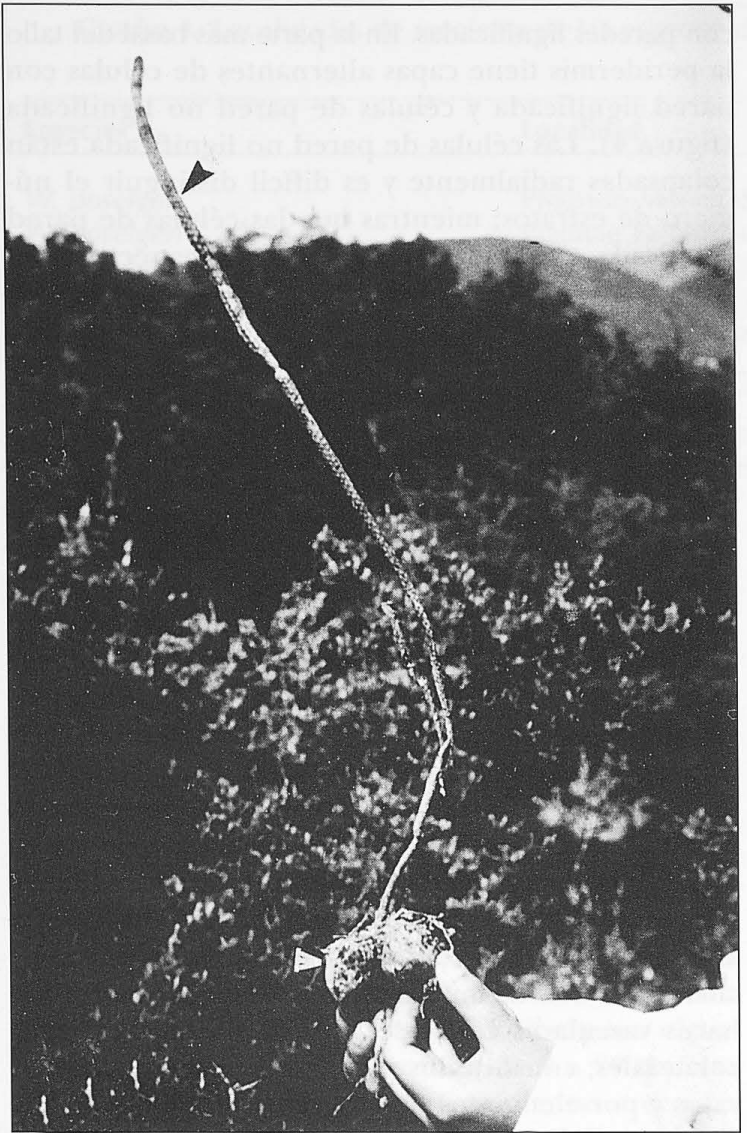

Figura 1. Planta de W. tamaulipensis colectada en el estado de Tamaulipas (Terrazas y Loza 319). Notar el esbelto tallo cilíndrico (flecha) de $51 \mathrm{~cm}$ en altura y las raíces tuberosas.

aprecia por la acumulación del floema colapsado sobre el floema secundario funcional, mientras que la acumulación del xilema secundario es escasa. Las células de mucílago son más abundantes en el clorénquima de la parte apical del tallo. Los cristales prismáticos son raros, sólo se observaron en algunas células parenquimáticas cercanas a la hipodermis en el tallo de un individuo de $W$. tamaulipensis. Se encontraron también cuerpos cristalinos que tienen forma esferoidal con estructuras concéntricas estriadas radialmente (figura 7). Estos cuerpos cristalinos tienen un diámetro que varía de 48 a $115 \mu \mathrm{m}$ en $W$. poselgeri y de 113 a $195 \mu \mathrm{m}$ en $W$. tamaulipensis (cuadro 2) y se observaron a lo largo del tallo con excepción de la parte apical de éste. En la parte basal del tallo, el aumento en espesor del cilindro vascular ocasiona que todas las células de la corteza se colapsen. Sin embargo, los vasos y el floema funcional de los haces vasculares corticales y los cristales esferoidales no se colapsan. Estos últimos son conspicuos y de tamaño relativamente grande.

Xilema primario. La estela consiste de un conjunto de 17 haces vasculares colaterales que se fusionan y forman un cilindro sólido a medida que se establece el crecimiento secundario. Cada haz vascular tiene de dos a ocho vasos y células de parénquima del xilema. Los elementos de vaso tienen paredes secundarias con engrosamientos helicoidales y placa de perforación simple con orientación generalmente transversal.

Floema. Está constituido por elementos de tubo criboso, células acompañantes y parénquima y no se observó esclerénquima en ningún individuo. Los elementos de tubo criboso tienen forma angulosa y placa cribosa simple casi transversal. Las células acompañantes presentan una forma triangular. A medida que el crecimiento secundario se establece, se distinguen bandas tangenciales de células de parénquima de una célula de grosor, alternadas con elementos de tubo criboso y células acompañantes. Las células del parénquima floemático son de forma casi cuadrada y conforme se expanden adquieren formas elongadas y acumulan extractivos color rojizo (figura 8). Este parénquima deja de expanderse en la parte basal del tallo y son notorios los elementos de tubo criboso y células acompañantes colapsados entre las bandas de parénquima floemático.

Medula. Tiene un diámetro de 40 a $48 \mu \mathrm{m}$ en la parte apical del tallo y en la parte basal del tallo fluctúa de 46 a $75 \mu \mathrm{m}$ en $W$. poselgeri, mientras que en $W$. tamaulipensis el diámetro en la parte apical fluctúa de 36 a $54 \mu \mathrm{m}$ y en la parte basal de 50 a $80 \mu \mathrm{m}$. La medula presenta células de parénquima que en su mayor parte son isodiamétricas y células de mucílago escasas. No se observaron haces vasculares ni algún tipo de inclusión.

Xilema secundario. La porosidad es difusa sin marcas de crecimiento distinguibles en W. tamaulipensis (figura 9) y anular en $W$. poselgeri. En esta última los anillos de crecimiento se distinguen por una disminución abrupta en el diámetro de las fibras en la madera tardía y por el tamaño y la agrupación de los vasos con parénquima vasicéntrico en la madera temprana. La densidad es de 7 a 13 vasos $/ \mathrm{mm}^{2}$ en $W$. tamaulipensis y en la madera tardía de $W$. poselgeri de 4 a 8 vasos $/ \mathrm{mm}^{2}$ (cuadro 2). Los vasos se arreglan entre 66 y $82 \%$ solitarios y el resto en grupos de 2 a 5 . Cerca de la medula disminuye el número y porcen- 
taje de agrupación. El diámetro tangencial varía de $15 \pm 3 \mu \mathrm{m}$ en $W$. poselgeri a $17 \pm 4 \mu \mathrm{m}$ en $W$. tamaulipensis. Los elementos de vaso tienen una longitud de $155 \pm 26 \mu \mathrm{m}$ en $W$. poselgeri y de $181 \pm 29 \mu \mathrm{m}$ en $W$. $t a-$ maulipensis (cuadro 2). En ambas la placa de perforación es simple con orientación generalmente transversal. Las punteaduras intervasculares son areoladas alternas y ocasionalmente pseudoescalariformes en $W$. poselgeri. También en esta especie, algunos vasos presentan depósitos obscuros que ocluyen su lumen.

Las fibras son libriformes, nucleadas con punteaduras simples más abundantes en las paredes radiales (figura 10). Además, las fibras presentan tendencia a un arreglo estratificado. La longitud varía de $313 \pm 50$ $\mu \mathrm{m}$ en $W$. poselgeri a $316 \pm 43 \mu \mathrm{m}$ en $W$. tamaulipensis. El diámetro es de $15 \pm 2 \mu \mathrm{m}$ en $W$. poselgeri y de $15 \pm 3$ $\mu \mathrm{m}$ en $W$. tamaulipensis, con un grosor de la pared de $3.0 \pm 0.6 \mu \mathrm{m}$ en $W$. tamaulipensis y de $3.6 \pm 0.6 \mu \mathrm{m}$ en $W$. poselgeri (cuadro 2). En un individuo de $W$. poselgeri se observó el lumen de algunas fibras ocluido por depósitos obscuros.

El parénquima axial es paratraqueal escaso o vasicéntrico asociado a la madera temprana en $W$. poselgeri. Las series parenquimáticas son de tres células. Se observan comúnmente grupos de células con paredes delgadas no lignificadas en la matriz de fibras. En estas zonas los vasos forman grupos de hasta cinco vasos y están rodeados de células parenquimáticas fusiformes.

El parénquima radial no se diferencia, por lo tanto la madera se reconoce como una madera sin radios. Al iniciar el crecimiento secundario las fibras que se diferencian en lugar de los radios son células cuadradas, tienden a estar dispuestas en hileras radiales y presentar paredes con un espesor menor que las paredes de fibras de las regiones fasciculares. Estas diferencias son imperceptibles con la mayor acumulación de xilema secundario.

\section{Descripción anatómica de la raíz}

En las raíces no tuberosas y tuberosas estudiadas sólo se observó crecimiento secundario. La estructura de la raíz no tuberosa es semejante a la del tallo y no se describe. Sin embargo, a continuación se detallan las características anatómicas de la raíz tuberosa.

Región central de la raíz. Está constituida por células de parénquima de forma isodiamétrica. El aumento en diámetro de la raíz se debe a un incremento tanto en número como en tamaño de las células parenquimáticas del metaxilema (figuras 11 y 12), asociado al incremento en espesor de la corteza primaria y el parénquima xilemático. En la región de máxima ex- pansión celular, la presencia de granos de almidón es más conspicua debido al tamaño y abundancia de éstos. Los granos de almidón son simples y de forma esferoidal. En esta región también son abundantes las células mucilaginosas (figura 12).

Corteza primaria. Está formada por células isodiamétricas de parénquima. Ocupa una proporción grande de tejido en la raíz de almacenamiento y también contribuye al aumento en diámetro de dicha raíz por un incremento en el tamaño de las células. En todo el tejido cortical se distribuyen células de mucílago y haces vasculares corticales. Al igual que en la región central de la raíz, las células de mucílago son más abundantes en la región de máxima expansión celular del tubérculo. Los haces vasculares corticales son colaterales y en algunos el floema está colapsado, semejantes a los presentes en el tallo.

Tejido vascular. Previo al aumento en diámetro de la raíz tuberosa los vasos están distribuidos en el centro del eje de la raíz y el cámbium vascular forma un cilindro continuo (figura 11). Sin embargo, una vez que se inicia la expansión, las células de parénquima xilemático entre las hileras vasculares radiales incrementan su número y tamaño con abundantes granos de almidón simples, grandes y de forma esferoidal. También en esta región parenquimatosa, hay abundantes células de mucílago, lo que dificulta apreciar la continuidad del cámbium (figura 12). Como se mencionó, con el aumento en diámetro de la raíz tuberosa, el xilema y el floema se restringen a hileras radiales (figura 12). Estas hileras radiales tienen de uno a cinco vasos de ancho y ocasionalmente las hileras de vasos son separadas por células de parénquima que también forman parte de las hileras radiales (figura 13). Los elementos de vaso tienen paredes secundarias con engrosamientos helicoidales y placa de perforación simple o raramente reniforme. El floema presenta una posición periférica a cada hilera radial de xilema (figura 13). Los elementos de tubo criboso y células acompañantes forman grupos separados por células de parénquima (figura 14). Comúnmente se observan regiones extensas de floema colapsado (figuras 11 y 12).

Peridermis. La peridermis tiene el mismo origen y estructura que en el tallo, sólo que el número de estratos celulares es mayor en la raíz tuberosa.

\section{Discusión}

La anatomía del tallo y de la raíz en las dos especies estudiadas de Wilcoxia son similares, sólo difieren en 


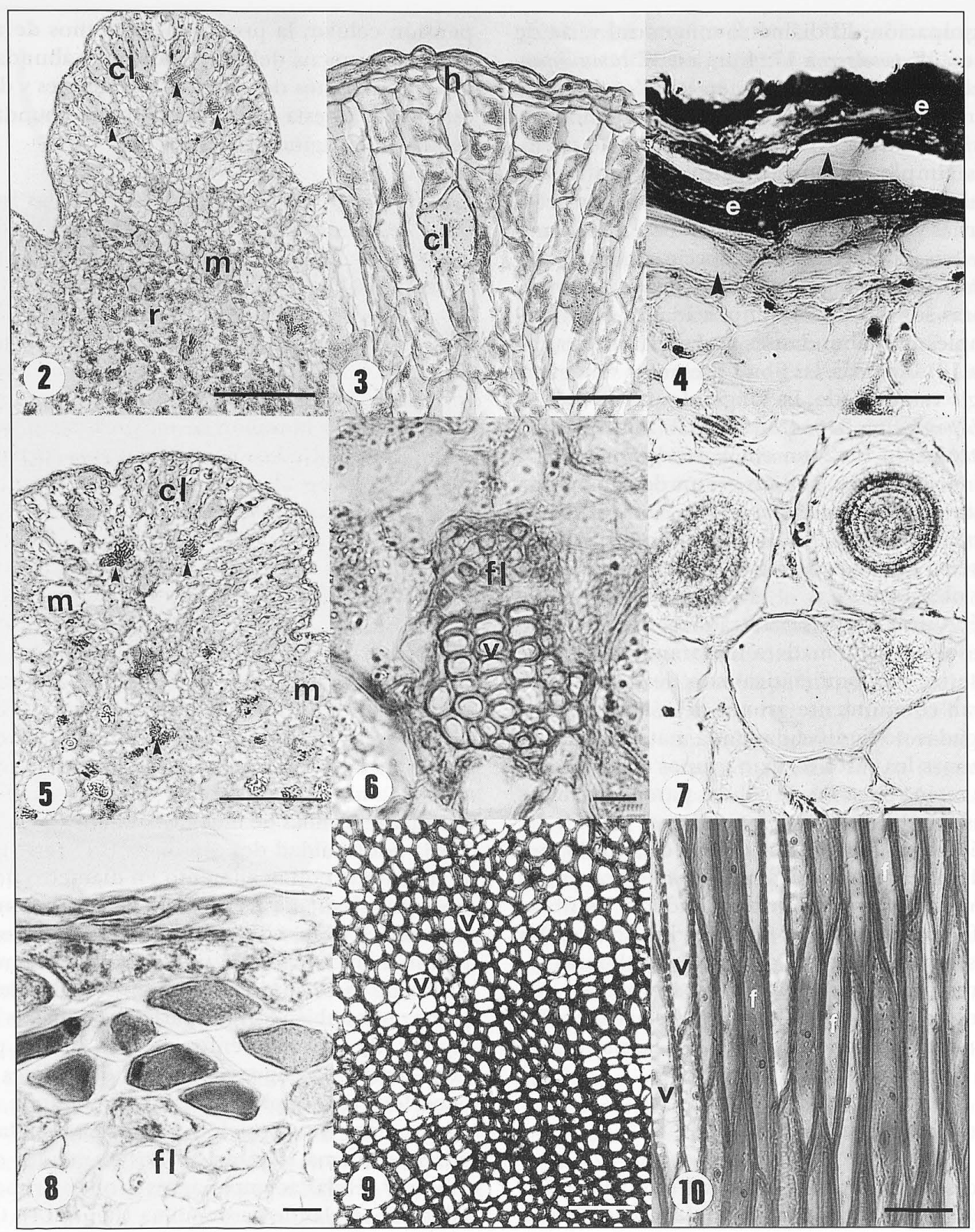

Figuras 2-10. Caracteres anatómicos del sistema dérmico, fundamental y vascular del tallo en Wilcoxia. 2-9 cortes transversales. 2. W. poselgeri. Epidermis y corteza primaria. Abundantes granos de almidón en el parénquima de reserva y haces corticales en el clorénquima (Flechas). 3. W. poselgeri. Epidermis lisa e hipodermis uniestratificada. Nóte el arreglo en hileras radiales de las células del clorénquima. 4. W. tamaulipensis. Peridermis con estratos de esclereidas que alternan con células de pared no lignificada (Flechas). 5. W. poselgeri. Corteza primaria con haces vasculares (Flechas) y células de mucílago. 6. W. poselgeri. Detalle de un haz vascular cortical de la parte media del tallo. 7. W. tamaulipensis. Cristales esferoidales de la corteza primaria. 8. W. tamaulipensis. Células de parénquima floemático con taninos. 9. W. tamaulipensis. Madera fibrosa con porosidad difusa y vasos solitarios o en grupos o hileras cortas. 10. W. tamaulipensis. Corte tangencial. Detalle de fibras libriformes con núcleo y con tendencia a un arreglo estratificado. $\mathrm{Cl}$ = clorénquima; $\mathrm{e}=$ esclereidas; $\mathrm{f}=$ fibras; $\mathrm{fl}=$ floema; $\mathrm{h}$ = hipodermis; $\mathrm{m}=$ células de mucílago; $\mathrm{r}=$ parénquima de reserva; $\mathrm{v}=$ vasos. Escala: $2,5=200 \mu \mathrm{m} ; 3=100 \mu \mathrm{m} ; 4,7,9,10=50 \mu \mathrm{m} ; 6,8=20 \mu \mathrm{m}$. 
el grosor de la cutícula y de la hipodermis, el tamano de los cristales esferoidales de la corteza primaria, el tipo de porosidad en el xilema secundario y en los caracteres cuantitativos longitud de los elementos de vaso y grosor de la pared de la fibra. Dichas diferencias así como la comparación de los caracteres anatómicos encontrados para el tallo y la raíz con los reportados para otros miembros de Cactoideae se discuten a continuación.

\section{Tallo}

Cutícula y epidermis. Los tallos de las especies estudiadas de Wilcoxia tienen una cutícula delgada, similar a otros géneros de Cactoideae como Acanthocereus, Echinocereus, Leptocereus, Neoevansia y Peniocereus (Gibson y Horak, 1978; Mauseth y Ross, 1988; Terrazas, observación personal). No obstante la cutícula de $W$. poselgeri es sumamente delgada en comparación con la de W. tamaulipensis. La epidermis es lisa, uniestratificada y con células rectangulares de pared delgada, semejante también a otros miembros de Cactoideae (Gibson y Horak, 1978). En vista superficial las células epidérmicas tienen paredes anticlinales onduladas o sinuosas, estas observaciones son semejantes a lo reportado por Eggli (1984) para las especies de Wilcoxia aquí estudiadas y varias especies de Cactoideae. Es importante señalar que los estomas son más abundantes en las depresiones de las costillas y es en esta región, donde se forma tardíamente la peridermis. Cabe destacar que esta distribución también se ha observado en algunas especies de Peniocereus (Hemenway y Allen, 1936; Terrazas, observación personal). La distribución de los estomas en las depresiones del tallo podría regular la tasa de transpiración en las plantas, además de mantener los estomas funcionales durante varios años.

Peridermis. En las especies estudiadas el felógeno se forma de las células epidérmicas y produce únicamente felema; por lo tanto, la peridermis es unidireccional. Este comportamiento no se ha reportado para otras cactáceas (Mauseth, 1996; Mauseth y Ross, 1988). El felema se caracteriza por presentar estratos de células con pared delgada no lignificada que alternan con esclereidas. Este arreglo estratificado también se ha observado en los géneros Leptocereus y Melocactus (Mauseth, 1989; Mauseth y Ross, 1988).

Hipodermis. En Cactoideae la hipodermis comúnmente es colenquimatosa, poliestratificada y con inclusiones ergásticas tales como cristales de oxalato de calcio o cuerpos de sílice (Gibson y Horak, 1978; Mauseth, 1996). Sin embargo, en Wilcoxia es de paredes del- gadas y uniestratificada. A pesar de que en ambas especies la hipodermis es uniestratificada, las células de W. tamaulipensis son más anchas, pero conservan su forma rectangular. La hipodermis uniestratificada no colenquimatosa podría estar relacionada con la talla corta y fuertemente leñosa de las especies.

Corteza primaria. A diferencia de otras especies, la región del clorénquima se distingue de la reservante, porque en las especies estudiadas las células más cercanas a la hipodermis tienden a formar hileras radiales. $\mathrm{El}$ arreglo de estas células permite la difusión de $\mathrm{CO}_{2}$ a través del aumento en espacios intercelulares y se denomina corteza en empalizada (Sajeva y Mauseth, 1991). Los haces vasculares corticales son colaterales, similares a los presentes en otras especies de Cactoideae (Gibson y Horak,1978; Mauseth y Ross, 1988; Mauseth y Sajeva, 1992). Al igual que en otros géneros de esta subfamilia (Gibson y Horak, 1978; Mauseth, 1989, Mauseth y Sajeva, 1992) no hay o es rara, la formación de xilema secundario en los haces corticales de Wilcoxia. Sin embargo, las observaciones de este trabajo muestran que hay acumulación continua de floema secundario y como consecuencia, las regiones de floema colapsado son más grandes en la parte basal del tallo. Mauseth y Sajeva (1992) señalan que los haces corticales posiblemente están involucrados en el transporte de agua, azúcares y fotosintatos. Las células de mucílago del tejido cortical son relativamente más abundantes y conspicuas en la parte apical del tallo de las especies estudiadas. Trachtenberg y Fahn (1981) mencionan que la diferenciación de células de mucílago se inicia en la proximidad del meristemo apical del tallo en otros géneros de Cactaceae y este puede ser el caso de Wilcoxia. Por otro lado, se menciona que las células idioblásticas de mucílago en especies de zonas áridas aparentemente funcionan como un mecanismo de retención de agua (Mauseth, 1988). Otra posible explicación es que éstas impiden el colapso total de las células de la corteza primaria en los periodos de deshidratación; favoreciendo la recuperación de la turgencia cuando el agua está disponible. Las especies de Wilcoxia también se distinguen por presentar en el tejido cortical cuerpos cristalinos de forma esferoidal con estructuras concéntricas estriadas radialmente conocidas como cristales esferoidales (Bailey, 1961; Metcalfe y Chalk, 1950). El tamaño de los cristales esferoidales separa a $W$. tamaulipensis de $W$. poselgeri. y es necesario saber si también se presentan y difieren en tamaño en las otras especies de Wilcoxia. Dichos cuerpos parecen estar asociados a las células mucilaginosas y no habían sido observados previamente en el género. Bailey (1961) consideró que estos cuerpos proba- 


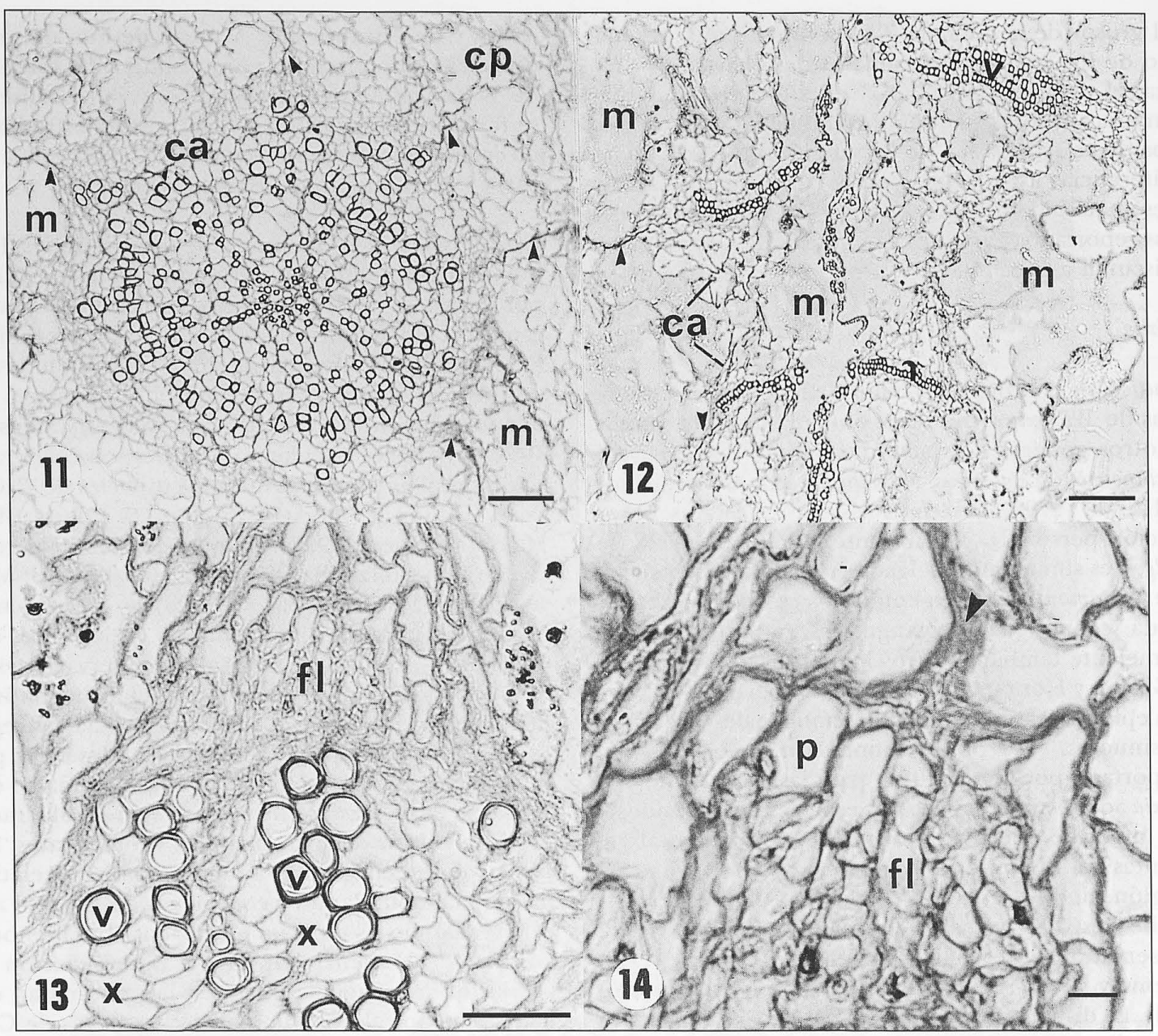

Figuras 11-14. Caracteres anatómicos de la raíz tuberosa en cortes transversales. 11. W. tamaulipensis. Región central del eje de una raíz tuberosa aún no ensanchada. El cámbium forma un cilindro continuo y comúnmente se observan regiones de floema colapsado (flechas). 12. W. poselgeri. El tejido vascular forma hileras radiales colaterales con floema colapsado distintivo (flechas) y xilema secundario. Las células de mucílago son abundantes en el parénquima que separa las hileras de tejido vascular y en la corteza primaria. 13. W. poselgeri. Detalle de tejido vascular. Hileras radiales de vasos con parénquima xilemático y floema. 14. W. poselgeri. Detalle de floema no colapsado con parénquima floemático y floema colapsado (Flecha). $\mathrm{Ca}=$ cámbium; $\mathrm{cp}=$ corteza primaria; $\mathrm{fl}=\mathrm{floema}$ no colapsado; $\mathrm{m}$ = célula de mucílago; $\mathrm{p}=$ parénquima floemático; $\mathrm{v}=$ vaso; $\mathrm{x}=$ parénquima xilemático. Escala: $11 \mathrm{y}$ $12=200 \mu \mathrm{m} ; 13=50 \mu \mathrm{m} ; 14=20 \mu \mathrm{m}$.

blemente son formas transicionales de cristalización de oxalato de calcio de las típicas drusas, y señala que la cristalización podría significar diferencias en la composición química. Es necesario, por tanto, una investigación detallada de la composición química de estos cuerpos cristalinos, así como de su variación a lo largo del año con objeto de evaluar si su presencia se asocia a los cambios de turgencia que ocurren en estas plantas.
Medula. En la mayoría de las cactáceas, la fusión tardía de los haces vasculares con crecimiento secundario favorece la expansión de la medula, ya que los haces se fusionan en un cilindro de madera sólido únicamente cuando termina dicha expansión (Gibson y Horak, 1978). En las especies de Wilcoxia ésto no ocurre, la ausencia de radios influye en la fusión temprana de los haces vasculares y limita el crecimiento de la medula. Como consecuencia la contribución de 
la medula a la turgencia de las especies es limitada. Además no se observaron haces vasculares medulares, lo cual coincide con lo reportado para otros géneros de Cactoideae (Gibson y Horak, 1978; Mauseth, 1993).

Floema. La ausencia de fibras sobre el protofloema es una característica distintiva para las especies de Wilcoxia estudiadas. Las especies de Cactoideae con fibras sobre el protofloema tienen comúnmente maderas fibrosas (Gibson y Horak, 1978; Mauseth, 1996); por lo que la ausencia de fibras, en las especies estudiadas con madera fibrosa, se considera un carácter diagnóstico. Además, en ambas especies, las células de parénquima forman bandas tangenciales que se alternan con los elementos de tubo criboso y las células acompañantes. La falta de información sobre el floema en otros miembros de Cactoideae no permite hacer comparaciones y concluir acerca del valor de la distribución del parénquima floemático en las especies estudiadas de Wilcoxia.

Xilema secundario. Las especies estudiadas de Wilcoxia forman un cilindro sólido de xilema constituido por fibras, vasos y parénquima axial. Las fibras son el elemento más abundante como ocurre en otras especies de Cactoideae (Gibson, 1973, 1978b), por lo que se consideran maderas fibrosas. Es importante resaltar que aun cuando ambas especies crecen en ambientes áridos, sólo $W$. poselgeri presenta porosidad anular. La porosidad anular únicamente se ha reportado para algunas especies de los géneros Echinocereus, Opuntia, Pseudomitrocereus y Rhipsalis (Gibson, 1973, 1978 a), por lo que se considera un carácter distintivo de $W$. poselgeri. Las diferencias encontradas para la longitud del elemento de vaso entre las especies pueden estar relacionadas con la altura de la planta como señala Gibson (1973).

La presencia de fibras con núcleos y granos de almidón es una característica también distintiva de las especies de Wilcoxia estudiadas. Este tipo de fibras es un estado de carácter con posible significado adaptativo (Fahn y Leshem, 1963; Wolkinger, 1969) y presumiblemente derivado (Dickison, 1986). El parénquima axial en las especies estudiadas es paratraqueal escaso. La escasez de parénquima axial se correlaciona con la presencia de elementos imperforados vivos (Dickison 1986); donde los últimos, además de proporcionar soporte mecánico tienen la capacidad de almacenamiento. Por otra parte, W. poselgeri tiene fibras con paredes de mayor grosor, pero con el diámetro de la fibra similar al de W. tamaulipensis, estos valores indican que $W$. poselgeri tiene un mayor peso específico.
Wilcoxia poselgeri y W. tamaulipensis se distinguen por la ausencia de radios al igual que W. albiflora (Gibson, 1973; Loza-Cornejo y Terrazas, observación personal). Desde el establecimiento del crecimiento secundario en las especies estudiadas se presentan fibras en lugar de radios en las áreas interfasciculares. Por lo tanto, el cámbium vascular tiene únicamente iniciales fusiformes. La ausencia de radios se considera una característica especializada del xilema secundario en otras especies de zonas áridas (Whalen 1987). Por otro lado, Barghoorn (1941) y Carlquist (1970) establecieron que las maderas sin radios son especializadas en asociación con otros caracteres como: presencia de fibras libriformes, parénquima axial del tipo paratraqueal, elementos de vaso con placa de perforación simple y punteaduras intervasculares alternas areoladas. Las especies de Wilcoxia estudiadas comparten los caracteres mencionados para maderas sin radios y se sugiere que tienen xilema secundario especializado con iniciales fusiformes cortas. Probablemente como señalan Carlquist (1975) y Whalen (1987) para especies de otras familias, la selección para una mayor resistencia mecánica fue un factor importante en la evolución del cámbium vascular de las especies de Wilcoxia. Gibson (1973) señala que las especies de Wilcoxia son plantas perennes subfrutescentes herbáceas, sin embargo observaciones de campo y de laboratorio muestran que son fuertemente leñosas tanto en el tallo como en las ramas, además de tener una alta capacidad de rebrote.

\section{Raiz tuberosa}

De acuerdo a Esau (1976) el carácter común a las raíces tuberosas es la presencia de abundante parénquima de almacenamiento. En el material estudiado, éste se encuentra en la región central de la raíz, la corteza primaria y el tejido vascular. En raíces tuberosas de Cactoideae estudiadas, la mayor parte de la suculencia se desarrolla en la corteza primaria (Gibson, 1978c) y en menor proporción en el parénquima del metaxilema (Gibson y Nobel 1986); mientras que en Opuntioideae, Pereskioidae y Nyctocereus serpentinus la mayor acumulación se presenta en el xilema secundario (Gibson, 1978c). Gibson (1978c) denomina al incremento de parénquima del metaxilema parenquimatización del protoestele y lo ha observado en Neoevansia diguetii y Peniocereus greggii. En el presente estudio se observó un proceso de parenquimatización similar; no obstante el parénquima xilemático entre las hileras radiales de xilema secundario, también contribuye a incrementar el diámetro de las raíces tuberosas.

En las raíces tuberosas estudiadas, el tejido vascular es escaso y se restringe a hileras radiales colatera- 
les estrechas como también ocurre en Peniocereus marianus y Wilcoxia albiflora (Gibson, 1973). Los elementos de vaso en la raíz tuberosa presentaron paredes con engrosamientos helicoidales y placa de perforación simple, y raramente, placa de perforación reniforme. Süss (1969) observó este tipo de placa de perforación en especies de Cereus, Eriocereus y Trichocereus. La presencia y abundancia de ambos tipos de placa de perforación es común en otras familias de dicotiledóneas (Carlquist, 1988); no obstante, se desconoce su significado funcional y evolutivo.

Los pocos estudios sobre anatomía de raíces tuberosas en Cactaceae muestran considerable variación en el origen del tejido de almacenamiento, por lo que coincidimos con Gibson (1978c), en la necesidad de realizar un mayor número de estudios sobre el desarrollo de este tipo de raíces, con objeto de entender los procesos de parenquimatización.

\section{Agradecimientos}

Esta investigación fue apoyada económicamente por CONACYT (Proyecto 0760-N a T.T.) y Cactus and Succulent Society of America. Agradecemos al M.C. Andrés Rodríguez Garay de la Universidad Autónoma Agraria Antonio Narro y al Ing. Agr. Jorge L. Jiménez Pérez, de la Universidad Autónoma de Tamaulipas, su valiosa ayuda en la recolecta de campo; al Dr. Héctor Hernández M. por facilitarnos ejemplares de W. tamaulipensis ssp. waldeisii; al Dr. Mario Sousa, curador del Herbario Nacional MEXU por permitirnos remover material de $W$. albiflora y $W$. poselgeri; al Sr. Miguel Vega por el trabajo fotográfico de cuarto obscuro; a la M.C. Jeannette Sofia Bayuelo Jiménez por la traducción del alemán y a tres revisores anónimos por las sugerencias para mejorar el manuscrito.

\section{Literatura citada}

Altesor A., Silva C. y Ezcurra E. 1994. Allometric neoteny and the evolution of succulence in cacti. Botanical Journal of the Linnean Society 114:283-292.

Arias M., S. 1993. Cactáceas: conservación y diversidad en México. Revista de la Sociedad Mexicana de Historia Natural 44:109-115.

Bailey I.W. 1961. Comparative anatomy of the leaf-bearing Cactaceae, III. Form and distribution of crystals in $\mathrm{Pe}$ reskia, Pereskiopsis and Quiabentia. Journal of the Arnold Arboretum 42:334-347.

Barghoorn E.S. 1941. The ontogenetic development and phylogenetic specialization of rays in the xylem of dicotyledons. III. The elimination of rays. Bulletin of the Torrey Botanical Club 68:317-325.

Barthlott W. y Hunt D.R. 1993. Cactaceae. En K. Kubitzki edr. The families and genera of vascular plants. SpringerVerlag, Berlín, 161-197.

Berlyn G.P. y Miksche J.P. 1976. Botanical microtechnique and cytochemistry. Iowa State University Press, Ames.

Boke N.H. 1951. Histogenesis of the vegetative shoot in Echinocereus. American Journal of Botany 38:23-36.

Boke N.H. 1959. Endomorphic y ectomorphic characters in Pelecyphora and Encephalocarpus. American Journal of Botany 46:197-207.

Bravo-Hollis H. 1978. Las cactáceas de México. 2da. ed. Universidad Nacional Autónoma de México. México.

Britton N.L. y Rose J.N. 1963. The Cactaceae. Vol. II. Dover Publications, Inc. New York.

Buxbaum F. 1973. Wilcoxia. H. Krainz, Die Kakteen. XII.

Carlquist S. 1970. Wood anatomy of insular species of Plantago and the problem of raylessness. Bulletin of the Torrey Botanical Club 97:353-361.

Carlquist S. 1975. Ecological strategies of xylem evolution. University of California Press, Berkeley.

Carlquist S. 1982. The use of ethylenediamine in softening hard plant structures for paraffin sectioning. Stain Technology 57:311-317.

Carlquist S. 1988. Comparative wood anatomy. Springer-Verlag. Berlín, Heidelberg.

Dickison W.C. 1986. Wood anatomy and affinities of the Alseuosmiaceae. Systematic Botany 11:214-221.

Eggli U. 1984. Stomatal types of Cactaceae. Plant Systematics and Evolution 146:197-214.

Esau K. 1976. Anatomía vegetal. 4a. ed. Omega. Barcelona.

Fahn A. y Leshem B. 1963. Wood fibres with living protoplasts. New Phytologist 62:91-98.

Flores-Vindas E.M. 1973. Algo sobre morfología y anatomía de semillas de Cactaceae. Tesis de Maestría en Ciencias. Colegio de Postgraduados. Chapingo, México.

Gibson A.C. 1973. Comparative anatomy of secondary xylem in Cactoideae (Cactaceae). Biotropica 5:29-65.

Gibson A.C. 1978a. Woody anatomy of Platyopuntias. Aliso 9:279-307.

Gibson A.C. 1978b. Architectural designs of wood skeletons in cacti. The cactus and Succulent Journal of Great Britain 40:73-80.

Gibson A.C. 1978c. Structure of Pterocactus tuberosus, a cactus geophyte. Cactus and Succulent Journal 50:41-43.

Gibson A.C. y Horak K.E. 1978. Systematic anatomy and phylogeny of Mexican columnar cacti. Annals of the Missouri Botanical Garden 65:999-1057.

Gibson A.C. y Nobel P.S. 1986. The cactus primer. Harvard University Press. Massachusetts.

Hemenway A.F. y Allen M.J. 1936. A study of the pubescence of cacti. American Journal of Botany 23:139-144

Hernández-García G. y García-Villanueva E. 1991. Anatomía comparada de óvulos y semillas de dos especies del género Mammillaria (Cactaceae). Boletín de la Sociedad Botánica de México 51:25-38. 
Hernández M.H. y Godínez H.A. 1994. Contribución al conocimiento de las cactáceas mexicanas amenazadas. Acta Botánica Mexicana 26:33-52.

IAWA Committee. 1964. Multilingual glossary of terms used in wood anatomy. Konkordia, Winterthur.

IAWA Committee. 1989. IAWA list of microscopic features for hardwood identification. IAWA Bulletin n.s. 10:219-332.

Lange M. 1995. Echinocereus sect. Wilcoxia-eine revision. Kakteen und andere Sukkulent 46:-133-144.

Mauseth J.D. 1988. Plant anatomy. Benjamin/Cummings Publishing. Redwood City, California.

Mauseth J.D. 1989. Comparative structure-function studies within a strongly dimorphic plant, Melocactus intortus (Cactaceae). Bradleya 7:1-12.

Mauseth J.D. 1993. Medullary bundles and the evolution of cacti. American Journal of Botany 80:928-932.

Mauseth J.D.1996. Comparative anatomy of tribes Cereeae and Browningieae (Cactaceae). Bradleya 14:66-81.

Mauseth J.D. y Ross R.G. 1988. Systematic anatomy of the primitive cereoid cactus Leptocereus quadricostatus. Bradleya 6:49-64.

Mauseth J.D. y Sajeva M. 1992. Cortical bundles in the persistent, photosynthetic stems of cacti. Annals of Botany 70:317-324.

Metcalfe C.R. y Chalk L. 1950. Anatomy of the dicotyledons. Clarendon Press, Oxford. 678-706.

Orozco C. 1987. Estudio anatómico de la epidermis de 7 especies de Opuntia de 20 localidades del Valle de México. Cactáceas y Suculentas Mexicanas 32:87-92.

Pimienta B.E., Loera-Quezada M. y López-Amezcua L. 1993.
Estudio anatómico comparativo en colectas del subgénero Opuntia. Agrociencia serie Fitociencia 4:7-21.

SAS Institute. 1989. SAS user's guide: statistics. Cary, North Carolina.

Sajeva M. y Mauseth J.D. 1991. Leaf-like structure in the photosynthetic, succulent stems of cacti. Annals of Botany 68:405-411.

Süss H. 1969. Uber reniforme Gefäbdurchbrechungen im Holz einiger Kakteen. Berichte der Deutschen Botanischen Gesellschaft 82:183-188.

Taylor, N.P. 1985. The genus Echinocereus. A Kew Magazine Monograph, Collingridge Books, Kew.

Terrazas, T. 1994. Wood anatomy of the Anacardiaceae: ecological and phylogenetic interpretation. Ph.D. dissertation. The University of North Carolina at Chapel Hill, North Carolina.

Trachtenberg S. y Fahn A. 1981. The mucilage cells of Opuntia ficus-indica (L.) Mill.-development, ultrastructure and mucilage secretion. Botanical Gazette 142:206-213.

Whalen M.A. 1987. Wood anatomy of the American Frankenias (Frankeniaceae): systematic and evoluionary implications. American Journal of Botany 74:1211-1223.

Wilkinson H.P. 1979. The plant surface (mainly leaf) Part I: Stomata. En: Metcalfe C.R. y Chalk L. Edrs. Anatomy of the dicotyledons. Vol.I. 2nd. ed. Clarendon Press, Oxford, 7-117.

Wolkinger F. 1969. Morphologie und systematische Verbreitung der lebeden Holzfasern bei Sträuchern und Bäumen. I. Zur morphology und zytologie. Holzforschung 23:135-144. 